\title{
RBF Neural Network-Based Frequency Band Prediction for Future Frequency Hopping Communications
}

\author{
Shengyan Zhu $\mathbb{D},{ }^{1}$ Yongjian Wang $\mathbb{D},{ }^{2}$ Jianbo Zheng $\mathbb{D}^{3}$, and Shupeng Wang $\mathbb{D}^{4}$ \\ ${ }^{1}$ Faculty of Quality Management and Inspection \& Quarantine Sanjiang Research Institute of Artificial Intelligence \& Robotics, \\ Yibin University, China \\ ${ }^{2}$ National Computer Network Emergency Response Technical Team, Coordination Center of China, China \\ ${ }^{3}$ Key Laboratory of Human-Machine Intelligence-Synergy Systems, Shenzhen Institute of Advanced Technology, \\ Chinese Academy of Sciences, China \\ ${ }^{4}$ Institute of Information Engineering, Chinese Academy of Sciences, Beijing, China
}

Correspondence should be addressed to Yongjian Wang; wyj@cert.org.cn, Jianbo Zheng; jb.zheng@siat.ac.cn, and Shupeng Wang; wangshupeng@iie.ac.cn

Received 30 January 2021; Revised 22 February 2021; Accepted 15 March 2021; Published 30 March 2021

Academic Editor: Wei Wang

Copyright (C) 2021 Shengyan Zhu et al. This is an open access article distributed under the Creative Commons Attribution License, which permits unrestricted use, distribution, and reproduction in any medium, provided the original work is properly cited.

On the basis of the chaotic features of the frequency hopping signal, frequency band prediction for frequency hopping signal can enhance the interference effect of the signal greatly. However, poor prediction accuracy often limits its development in the military field. Therefore, for the sake of enhancing the frequency band prediction accuracy of frequency hopping signal, this paper studies the radial basis function (RBF) neural network frequency hopping signal frequency band prediction model based on the gradient descent method and improved the particle swarm optimization algorithm, respectively. The former uses a step-by-step algorithm to optimize the center value and weight so that the network can find the most suitable initial state. Then, the clustering selection optimization algorithm is employed to optimize the central value. In addition, it optimizes the weight by using a gradient descent method of the optimal learning rate. The latter optimizes the structure of the RBF neural network through the combination of the subtractive clustering algorithm and improved the particle swarm optimization (PSO) algorithm. Simulation results demonstrate that the gradient RBF algorithm model performs better in terms of accuracy, but time efficiency is lower, while the PSO-RBF algorithm has better time efficiency.

\section{Introduction}

The purpose is to effectively combat the enemy's communication system or protect the security of our army's communication system in the modern military battlefield. In addition to the traditional physical attack accident, we can also attack the enemy's communication system by means of signal interference. Network confrontation has become the main mode of enemy-friend warfare. Its main form of confrontation is exploiting a security flaw in an enemy network system to invade the enemy network and reduce and destroy the use efficiency of the enemy network [1]. Therefore, we need to understand the enemy's communication frequency band, and if we interfere and strike arbitrarily without knowing anything, it will also interfere with our communication system. As a result, it is necessary to predict the communication frequency band of the enemy. Through the prediction of the frequency band of frequency hopping communication, we can understand the enemy communication frequency band and attack effectively. Therefore, the prediction of the frequency hopping signal is the most important work before the specific strike.

In the traditional conventional frequency hopping technology, both sides of the communication carry out synchronous hopping according to the agreed frequency hopping strategy in the process of communication to awaken the 
reliable transmission of data information [2]. However, with the increasing intensity of modern electronic warfare, the spectrum resources are becoming more and more limited, which leads to the intensification of mutual interference among all kinds of communication equipment and the continuous upgrading of the interference technology of the interference party. The traditional frequency hopping technology cannot meet the antijamming needs of modern electronic warfare. Therefore, based on conventional frequency hopping, many experts and scholars worldwide raise the communication technology of adaptive frequency hopping (AFH) [3]. The core technology is monitoring the channel in real time, carrying on the link quality analysis (LQA) and feeding the evaluation information back to the other side of the communication. Then, the two sides communicate adaptively at the same time according to a certain strategy. Adaptive frequency hopping technology dramatically enhances the antijamming ability of the communication system. The common adaptive frequency hopping techniques include frequency point adaptive frequency hopping technology, power adaptive frequency hopping technology, frequency hopping rate adaptive frequency hopping technology, and the adaptive frequency hopping technology combined with them.

Nowadays, the existing neural network researches mainly focus on three aspects: network structure and optimization, learning and training algorithm, and practical application [4]. According to the real-time performance of network applications, these researches are separated into two fields: static network research and dynamic network research. The back propagation network (BP network) is the most extensively applied network model. It has a strong biological background because of its excellent input-output mapping characteristics. The BP network has a strong advantage in multivariable function approximation. Although it can achieve global optimization in theory, the optimization easily sinks into local minimum due to the algorithm restriction. The radial basis function network not only has the biological background but also coincides with the function approximation theory and is also suitable for multivariable function approximation. The theoretical basis of the orthogonal polynomial function network is relatively perfect, but for the nonlinear modeling and prediction of complex problems, the magnitude of network nodes often augments rapidly [5]. The advantage of the spline function network is that it only needs local information when learning; so, it has obvious advantages in the parallelism and convergence velocity of the algorithm, but because the subarea network separation in its definition domain is extraordinary complex, thus increasing the difficulty of practical application. The existing dynamic network research mainly focuses on real-time control, which requires the designed network to be simple in structure and fast in convergence. Typical dynamic networks include Hopfield network, adaptive resonance theory (ART) network, and dynamic recursive network [6]. The dynamic network is a single-layer network in the network structure, realizing the system's complex behavior control and simulation with less network structure overhead because of the internal feedback connection. So, it is more suitable for non- linear dynamic system identification and control and other fields.

In view of the existing network vulnerability analysis methods, the important differences of nodes in the network and the relationship between network nodes are often ignored. The research challenges of prediction frequency are listed as follows: (1) The prediction accuracy of frequency hopping signals with interference is strictly requested in the military. Meanwhile, the applied prediction scheme must have a high operability. (2) Considering that the military scenario has a strict requirement on the timeliness of applications, it is rather a challenge to reduce the running time of the applied algorithm while ensuring accurate decisions are made. (3) Limited spectrum resources aggravate the interference between communication devices. Designing a method overcoming the interference among devices while ensuring network efficiency is necessary. In the network vulnerability analysis, this project considers the communication characteristics of the node itself and the standing and influence of the network node structure from the multidimensional point of view of time and space. Finally, the fragile node is determined to provide a prerequisite for the frequency band selection of interference targets. The main contributions of this paper are summarized as follows:

(1) Compared with the interference to the enemy signal in the military field, this scheme predicts the frequency band of the frequency hopping signal from the point of view of the nature of the frequency hopping signal. It not only enlarges the precision of the interference but also is more convenient in practical operation

(2) The clustering selection optimization algorithm is introduced into the radial basis function neural network (RBF neural network) to predict the frequency band of frequency hopping communication. It is improved based on $k$-means and has higher accuracy than the $k$-means algorithm. It provides convenient conditions for the second step prediction and reduces the amount of computation. The concept of the optimal learning rate is introduced into the traditional gradient descent method, which makes the optimization process more efficient and convenient. The optimal learning rate is calculated by the least mean square matrix algorithm, which effectively reduces the running duration of the algorithm

(3) The second scheme applies the improved particle swarm optimization algorithm to the RBF neural network and uses a subtractive clustering algorithm to optimize the number of RBF network centers. The proposed scheme in this paper availably raises the overall efficiency of the network and the accuracy of frequency hopping communication band prediction

The remaining work of this paper is organized as follows: Section 2 introduces the frequency hopping signal prediction scheme based on the RBF neural network proposed, Section 3 


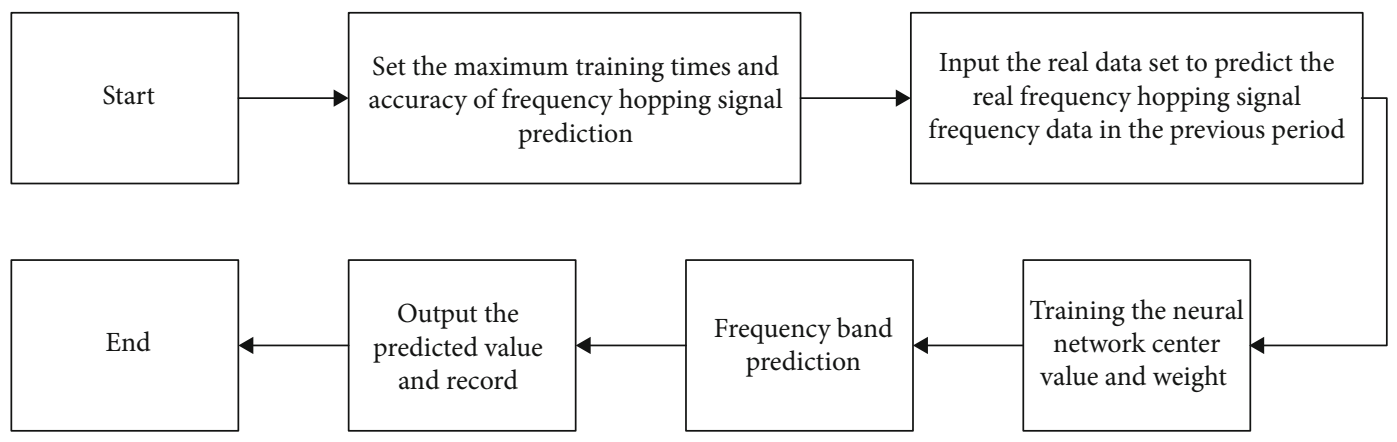

FIGURE 1: The RBF neural network prediction of frequency hopping signal flow diagram.

introduces the structure of RBF neural network based on the improved particle swarm optimization and subtractive clustering algorithm, Section 4 explains the experimental results, and Section 5 summarizes the full-text work.

\section{Frequency Band Prediction Model of Frequency Hopping Signal Based on the Gradient Descent Method}

This section proposes an RBF neural network prediction model based on an improved gradient descent method due to the existing problem of low accuracy of prediction models. It combines the clustering algorithm and the optimal learning rate to predict the frequency band of the frequency hopping signal.

2.1. Design Overview. In the RBF neural network prediction model, this scheme regards the current signal frequency and the signal frequency of the next time unit as the input and output in the RBF neural network [7], respectively. This prediction process is adaptive and is carried out gradually. In the specific prediction process, the network is constantly optimizing according to the actual value of the predicted signal. The number of signals in this process always remains the same, that is, when the next signal is optimized, the first signal is removed, and when the next signal is predicted, the first two signals are removed. In this way, a requirement of always keeping the same data optimized in the network is achieved. This approach can reduce the computing time and improve the optimization efficiency of the entire network. The method of real-time prediction of the network structure can greatly improve the accuracy of the network to achieve the experimental goal. Figure 1 shows a schematic diagram of the specific RBF neural network prediction process.

According to Figure 1 above, the RBF neural network's prediction model needs to go through the following steps: First, set the accuracy of the predicted model to achieve higher prediction accuracy than the existing scheme, which is set as $90 \%$. Next, we need to obtain a certain number of frequency hopping signal transmission frequency bands as the training set of the algorithm and then use the generated data set to optimize the structure of the entire network and determine the number, size, and weight of the center. Then, the frequency band prediction of the frequency hopping signal can be realized.
In the prediction process of the RBF neural network, training the central value and weight of the neural network is the key step in this scheme, and it is also the key research link in this section. The training of the central value and weight directly determines the quality of the RBF neural network prediction model. This section proposes a scheme based on a clustering selection optimization algorithm and gradient descent method, which constructs an RBF neural network prediction model to achieve an accurate prediction of frequency hopping signal frequency bands.

The prediction model that had been put forward in this section is divided into two steps in the optimization design of the model [8] according to the gradient-RBF neural network. The first step is to optimize the selection of the central value in the RBF neural network, and the main application is a new cluster selection optimization algorithm. Compared with the commonly used $k$-means clustering selection optimization algorithm, it will make the size of the center value more accurate, thereby reducing the time required for the second step, improving the work efficiency of the network, and improving the accuracy. The second step is the optimal selection of weights. This section uses the gradient descent method and the least mean square algorithm to convert the learning rate in the gradient descent method into the optimal learning rate so that the efficiency of the entire neural network will be significantly improved, and the signal frequency prediction will be more accurate.

2.2. Selection of the Number of the Center $c$ of the Hidden Layer of the RBF Neural Network. This program introduces the RBF neural network prediction method, which is used to predict the frequency of the frequency hopping signal in the frequency hopping communication. Due to the particularity of the RBF neural network structure, it is necessary to construct a reasonable network structure before predicting the frequency of the frequency hopping signal to achieve the purpose of accurate prediction. Therefore, how to find the optimal structure has become the most important issue in this scheme. The main idea of selecting the center is to make the center point as the center of a certain area, and the number of centers is required to be appropriate.

The pseudorandom code irregularly controls the frequency of the frequency hopping signal, but it may be concentrated in a certain area at different times [9]. Therefore, choosing the center value in these several areas will make 


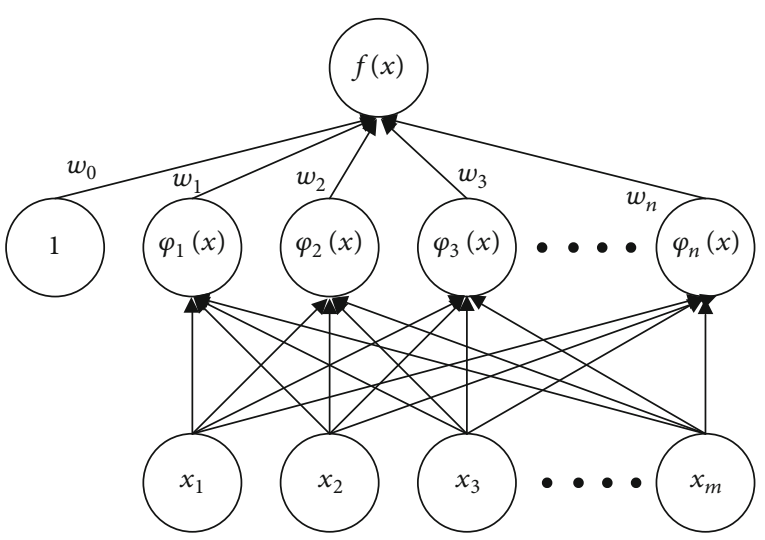

FIGURE 2: The input-neuron-output diagram of the RBF neural network.

the neural network structure more optimized and simplify the training process during the training process. The following scheme introduces an improved algorithm based on the $k$ -means clustering selection algorithm. This algorithm is more suitable for one-dimensional input of frequency hopping signal frequency, and its calculation time is shorter, and the accuracy is higher.

After comparing the radial basis functions mentioned above, this scheme selects the Gaussian function as the radial basis function of the RBF neural network [10]. Because the Gaussian function decreases monotonously from the center to both sides, its response is locally limited. There will be a local response only at a position close to its center. The Gaussian function is similar to the biological neuron in the RBF neural network and the linear summation weighted by $N$ Gaussian functions as the final output. Thus, the RBF neural network transformed the original nonlinear problem into the current linear problem [5]. The functional equation $f(x$ )$=w_{0}+\sum_{i}^{n} w_{i} \varphi\left(\left\|x-c_{i}\right\|\right)$ is the mapping equation from input to output, where $c_{i}(1 \leq i \leq n)$ denotes the center of the RBF neural network, and norm $\|\bullet\|$ represents the Euclidean distance and $w_{i}(1 \leq i \leq n)$ is the weight of the linear summation. $x$ and $w_{o}$ represent the input vector and deviation, respectively. The Gaussian function is selected as the radial basis function $\varphi(\cdot)$. Figure 2 shows a schematic diagram of its structure.

For the selection of center $c$, this section introduces an improved cluster selection optimization algorithm based on the $k$-means clustering algorithm, which has a faster convergence speed than the $k$-means algorithm, and the accuracy of optimizing the center value is higher.

In this learning method, the center is determined by selforganizing learning, which can be moved. The selection of the center can use the mean clustering selection optimization algorithm. This is an unsupervised learning method, and the center changes with the number of samples. The following are the detailed steps:

(1) Initialize cluster center $c_{i}(1 \leq i \leq n)$. If the value of $n$ is increasing, the accuracy is also improved, but the amount of calculation will increase [11]. Select $n$ samples from the input sample $x(1,2, \cdots, m)$ as cluster centers. ( $n$ is given in advance, according to the characteristics of the frequency hopping signal, $n$ is given as 50)

(2) Group the input samples according to the nearest neighbor rule, assign $x(1,2, \cdots, m)$ to the center $c$; $\theta_{n}$ is the cluster set of the input samples and meets $d=d_{\min }=\left\|\mathrm{x}_{m}-c_{i}\right\|$, and $j$ is the number of elements in each cluster set

(3) Calculate the average distance $\bar{d}=(1 / j) \sum_{1}^{i}\left\|x_{j}-c_{i}\right\|$ of each center $c_{i}$ and the average distance $\bar{D}=(1 / m)$ $\sum_{1}^{m}\left\|x_{m}-c_{i}\right\|$ of the entire system (each input $x_{m}$ corresponds to its cluster center)

(4) Find $c_{a}$ corresponding to $\bar{d}_{i \max }$ and randomly select another center $c_{a}^{\prime}$, if it is $\bar{D}_{i}^{\prime} \leq \bar{D}_{i}$, keep $c_{a}^{\prime}$; otherwise, keep $c_{a}$

Repeat steps 3 and 4 , when the random center $c^{\prime}{ }_{a}$ always satisfies $\bar{D}_{i}^{\prime} \leq \bar{D}_{i}$, and the optimization is completed, and the cluster center $c_{i}$ is output. The width of the RBF network is $\sigma=d / \sqrt{N}$, where $d$ represents the maximum distance between all centers and $N$ represents the number of centers.

2.3. Improved Gradient Descent Method and Implementation Scheme. After the center optimization is selected, the weights are optimized by the gradient descent method. In this scheme, the Gaussian function and matrix are used to calculate the actual output value $\hat{y}$ of the network, and $y, t$, and $w$ denote the actual output value of the network, the number of iterations, and the weight, respectively. Herein, $e(t)$ represents the error function, $E(t)$ is the cost function, and $\eta(t)$ is the learning efficiency function.

The update function of the weight is

$$
w(t+1)=w(t)-\eta(t) \frac{d E(t)}{d t} .
$$

By using the least mean square algorithm, we can obtain

$$
\varphi\left(\left\|x-c_{i}\right\|\right)=\exp \left(-\frac{1}{2 \sigma^{2}}\left\|x-c_{i}\right\|^{2}\right)(i=1,2, \cdots, n),
$$

where $n$ is the center number, and $m$ is the total number of input samples. In this experiment, we assume $n$ is 50 .

$$
\begin{gathered}
\varphi_{j i}=\varphi\left\|x_{j}-c_{i}\right\| j=1,2 \cdots, m, \\
\phi \omega=y \omega=\left(\omega_{1}, \omega_{2}, \cdots, \omega_{n}\right)^{T} .
\end{gathered}
$$

The cost function can be obtained by

$$
E(t)=\frac{1}{2} \sum_{j=1}^{m}\left[y_{j}-y \wedge(t)\right]^{2}=\frac{1}{2} \sum_{j=1}^{m} e_{j}^{2}(t) .
$$

By using the gradient descent method for function $E(t)$, 
we can obtain

$$
\begin{gathered}
\nabla E(t)=\sum_{j=1}^{m} e_{j}(t) \frac{\partial e_{j}(t)}{\partial \omega(t)}=-\sum_{j=1}^{m} e_{j}(t) \frac{\partial\left[\varphi_{j} \bullet \omega(t)\right]}{\partial \omega(t)}, \\
\nabla E(t)=-\sum_{j=1}^{m} e_{j}(t) \varphi_{j}^{T} .
\end{gathered}
$$

The variable for the $t$ th learning weight is $\Delta \omega(t)$.

$$
\begin{gathered}
\Delta \omega(t)=\omega(t+1)-\omega(t), \\
\Delta \omega(t)=-\eta(t) \nabla E(t-1)=\eta(t) \sum_{j=1}^{m} e_{j}(t-1) \varphi_{j}^{T} .
\end{gathered}
$$

The optimal learning rate is to use the learning rate in the gradient descent method to achieve the highest efficiency through matrix calculation [12]. The scheme is listed as follows:

$$
\Delta e(t)=e(t)-e(t-1)=-\Delta \hat{y(t)}=-\eta(t) \phi \Delta \omega(t)=-\eta(t) \phi \phi^{T} e(t-1)
$$

$$
\begin{gathered}
e(t)=e(t-1)-\eta(t) \phi \phi^{T} e(t-1), \\
E(t)=\frac{1}{2} e^{T}(t) e(t)=\frac{1}{2}\left[e(t-1)-\eta(t) \phi \phi^{T} e(t-1)\right]^{T} \bullet\left[e(t-1)-\eta(t) \phi \phi^{T} e(t-1)\right] .
\end{gathered}
$$

From the above derivation, the optimal learning rate can be obtained:

$$
\eta^{*}(t)=\nabla e(t)=e(t)-e(t+1)=\frac{e^{T}(t-1) \phi \phi^{T} e(t-1)}{e^{T}(t-1) \phi \phi^{T} \phi \phi^{T} e(t-1)}
$$

Specific steps are as follows:

(1) We need to get the number of hidden layer nodes $n$ through the above method and use the mean cluster selection algorithm to find the center $c$ and the width $\sigma$. Set learning accuracy rmse* and the maximum number of iterations max $t$

(2) Set the weight of the output layer and hidden layer to $w_{0}=\left(\begin{array}{c}1 \\ \vdots \\ 1\end{array}\right)$ and calculate $E(t)=$ rmse

(3) Let $\eta(t)=\eta^{*}(t)$ and $w=w+\eta(t) \phi^{T} e(t-1)$ iterate

(4) If rmse $\geq$ rmse $^{*}$ or $t \leq \max t$, repeat step 3; otherwise, stop and output $\hat{y}$ and $t$

The above optimization scheme can obtain the center, weight, and width of the RBF neural network through optimization training and learning [13]. When testing the data, the obtained data can continue to be input into the network structure as a sample, and the above steps are repeated. In this way, when the test data is more, the network structure is more accurate, and the measurement result is more accurate.

\section{Frequency Band Prediction Model of Frequency Hopping Signal Based on the RBF Neural Network Based on Improved Particle Swarm Optimization (PSO-RBF Algorithm)}

In view of the limitations of the above schemes in the military field and relatively speaking, the algorithm simulation time is long, and a global prediction scheme is proposed to predict the frequency band of frequency hopping signals in this section. This scheme uses the subtractive clustering algorithm and improved the particle swarm optimization (PSO) algorithm. We propose an RBF neural network prediction model based on improved PSO and optimize the center value and weight of the network to achieve the purpose of frequency hopping signal frequency band prediction.

3.1. Design Overview. In the previous section, the center and weight of the hidden layer in the RBF neural network are optimized by the step-by-step method. To improve the accuracy of the scheme as a whole, the accuracy of each step is high, which leads to too many iterations. Therefore, this section applies the improved PSO algorithm to the RBF neural network to predict the signal frequency band in the frequency hopping communication [14]. The prediction model proposed in this section optimizes the structure of the RBF neural network through real data, compares the predicted value with real value, and reoptimizes the adaptive prediction process of the network mechanism with real value as the training sample. Figure 3 shows a schematic diagram of the specific forecasting process.

Figure 3 above shows the prediction diagram, which is similar to the prediction process mentioned in Section 2. Some improvements are made on the optimization of the center, width, and weight of the RBF neural network. Data flow in this prediction process represents the real FM signal transmission frequency. The real value is taken as the training sample of the prediction model, which is optimized by the scheme in this section. By constantly adjusting the parameters, we can finally achieve the purpose of optimizing the RBF neural network construction.

In this scheme, the improved PSO algorithm is applied to the RBF neural network to predict the frequency band of the frequency hopping signal model. PSO is a random search algorithm that simulates biological activities in nature and is a branch of evolutionary computing. It finds the optimal solution through the cooperation mechanism in the population and is extensively used in various engineering optimization problems. The RBF neural network prediction model applies the improved PSO algorithm, which regards the center, width, and weight of the RBF neural network as a threedimensional optimization structure, and optimizes the prediction model continuously through the parameters and 


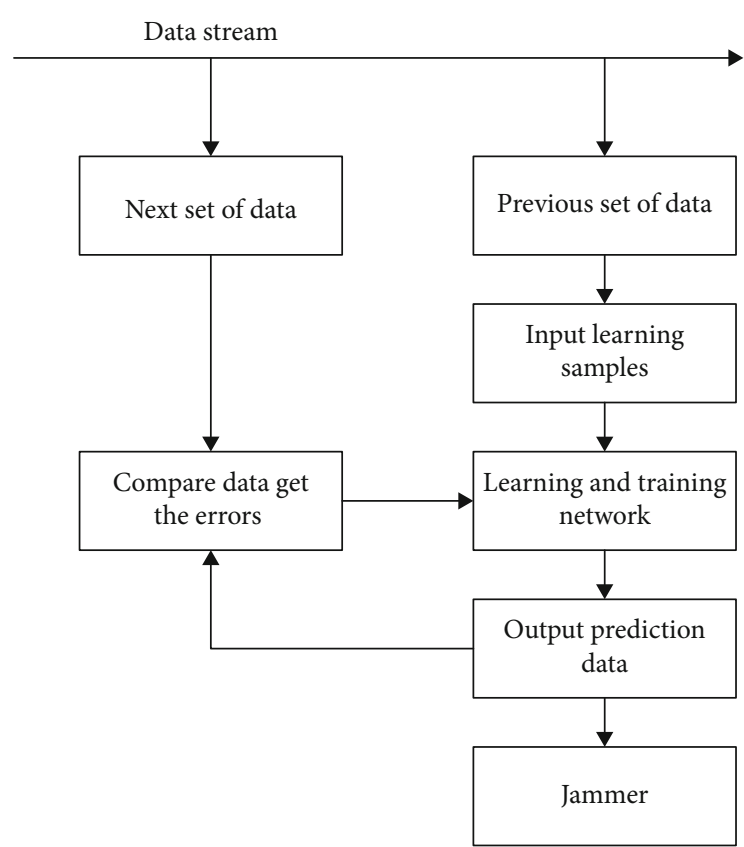

FIGURE 3: The data processing flow diagram of the prediction system.

fitness function of the particle swarm [15]. The step of optimizing the speed and position of particles is the most important link in this scheme. Combined with the advantages of the above two, the PSO algorithm is used to optimize the central parameters of the basis function. While using the subtractive clustering algorithm to determine the number of centers of the radial basis function, the PSO algorithm is used to adjust the connection weight between the hidden layer and the output layer of the network to achieve the overall optimization effect of the RBF neural network [16].

3.2. The Center Number of the RBF Neural Network Is Determined by the Subtractive Clustering Algorithm. The Gaussian function constitutes the vector basis, and several vector bases constitute the hidden layer of the RBF neural network [17], which is a method of approximating nonlinear functions by linear functions. In this section, the radial basis function used in the hidden layer of the RBF neural network is the Gaussian function, and the transmission frequency of frequency hopping signal in frequency hopping communication is the nonlinear function. In this scheme, the accuracy of the prediction model is directly determined by the number of radial basis functions in the hidden layer of the neural network. Under normal circumstances, the more basic functions, the higher the accuracy of the algorithm and then the more iterations of the algorithm [18]. Therefore, the key to this prediction model is that the count of hidden layer centers needs to be determined.

The subtractive clustering algorithm is a relatively effective clustering method to determine the number of basis function centers. The calculation principle of the subtractive clustering algorithm is to select the first central point from the position with the highest density in a group, get the first central point, subtract this point and the points near this point, and then repeat this process, and so on; until the current highest density is met. The specific algorithm is as follows.

Consider normalizing the data into $p$ data points $\left(x_{1}, x_{2}\right.$ $\left., \cdots, x_{p}\right)$ in an $n$-dimensional unit of hyperspace [19], the following formula gives the density at data point $x_{i}$ :

$$
D_{i}=\sum_{i=1}^{p} \exp \left[-\frac{\left\|x_{i}-y_{j}\right\|^{2}}{\gamma_{a} / 2}\right] \text {. }
$$

After calculating the density of each data point, we chose the point with the highest density as the first cluster center and denoted its density as $D_{c}$. The updated formula for the density of each data point is

$$
D_{i}=D_{i}-\sum_{i=1}^{p} \exp \left[-\frac{\left\|x_{i}-x_{c 1}\right\|^{2}}{\gamma_{a} / 2}\right]
$$

After the density of each point is updated, we select the next clustering center and modify its density again [20]. This process is repeated until the current maximum density $D$ is far less than the initial maximum density, and the formula is as follows:

$$
\frac{D_{\max }}{D_{c 1}}<\lambda
$$

Thus, the clustering ends, and the number of clustering centers obtained is equal to the number of centers of the basis function.

\subsection{Implementation of Improved PSO in the RBF Neural Network}

3.3.1. Fitness Selection Based on Frequency Hopping Signal. In this scheme, the fitness function selects the error function of the whole system, which can show the quality of the system more intuitively and has more intuitive significance for the optimization of the internal structure of the system.

In the PSO algorithm, a particle corresponds to a feasible solution. First, we code the particle, which includes the center value and width of the basis function, particle velocity, and fitness [21]. According to the subtractive clustering algorithm, suppose $m$ centers are determined and each center is $k$-dimensional, then the position of the particle is $m \times(k+$ 1)-dimensional, the velocity of it is also $m \times(k+1)$-dimensional, $\sigma_{i}$ represents the width of the $i$ basis function, and $f_{i}$ is the fitness of the $i$ individual. Formulas (17) and (18) show the fitness function:

$$
\begin{gathered}
f_{i}=\frac{1}{R_{i}}, \\
R_{i}=\frac{1}{M} \sum_{k=1}^{m}\left(y_{k}-y \wedge_{k}\right)^{2} .
\end{gathered}
$$


TABLE 1: Initialization values of PSO-RBF algorithm.

\begin{tabular}{lc}
\hline Parameter & Value \\
\hline Number of iterations $G$ & 20 \\
Particle dimension $n$ & 15 \\
Population size $m$ & 20 \\
Algorithm parameters $w$ & 0.1 \\
Algorithm parameters $c 1$ & 2 \\
Algorithm parameters $c 2$ & 2 \\
\hline
\end{tabular}

3.3.2. Selection of the Update Function of Improved PSO. A random particle swarm is generated by the PSO algorithm, and each particle is given a random velocity. During the flight, the speed of the particles is dynamically adjusted by the pulling force of their own and companions' flight experience, and the whole group can fly to a better search area [22]. Suppose that the search space is $N$-dimensional, the total number of particles is $Z$, the position of the $i$ particle in the $N$-dimensional space is expressed as $x_{i}=\left(x_{i 1}, x_{i 2}, \cdots, x_{\mathrm{iN}}\right)$, and the flight speed is expressed as $v_{i}=\left(v_{i 1}, v_{i 2}, \cdots, v_{\mathrm{iN}}\right)$. The optimized objective function assigns a fitness value to each particle. Each particle is searched in the solution space immediately following the current optimal particle. In iterations, each process is not completely random, and if we find a better solution, we can use it as a basis for finding the next solution. Each time the particle updates itself, it tracks two "extremes" in the iteration: one is the individual extreme point [23], that is, the best solution found by the particle itself, whose position is expressed as pbest; the other is the global extreme point, which is the best solution found by the whole population at present, and its position is expressed as gbest. When the two best solutions are found, the velocity and position of particle will be updated according to formula (19):

$v_{i d}^{k+1}=w v_{i d}^{k}+c_{1} \operatorname{rand}_{1}^{k}\left(\right.$ pbest $\left._{i d}^{k}-x_{i d}^{k}\right)+c_{2} \operatorname{rand}_{2}^{k}\left(\right.$ gbest $\left._{i d}^{k}-x_{i d}^{k}\right)$,

where $v_{i d}^{k}$ denotes the $d$-dimensional component of the flight velocity in the $k$ iteration of the $i$ particle, and $x_{i d}^{k}$ represents the $d$-dimensional component of the position in the $k$ iteration of the $i$ particle [24]. pbest $_{i d}^{k}$ denotes the position of the individual extreme point of the particle $i$ in the $d$-dimension. gbest ${ }_{i d}^{k}$ denotes the position of the global extreme point of the whole swarm in the $d$-dimension. $w$ stands for inertia weight, and $c_{1}$ and $c_{2}$ are learning factors. The maximum step size of the global best particle and the individual best particle flying in the direction of the global best particle and the individual best particle is adjusted by them severally. General $\operatorname{order} c_{1}=c_{2}=2$ andrand 1are stochastic numbers among [0, $1]$.

Different from the application of the traditional particle swarm optimization algorithm, the prediction model of the RBF neural network regards the radial basis function in the hidden layer of the neural network as a particle. The center value, width, and weight are regarded as particles that need to be searched in the three-dimensional solution space. Therefore, the value of $d$ in this scheme is 3 . pbest ${ }_{i d}^{k}$ represents the best position of the predicted value of this time in the training sample, and gbest ${ }_{i d}^{k}$ represents the best position of the predicted value of all training samples up to the current time. In this training model [25], each radial basis function has a memory function and records its center value and width, as well as its weight connected to the output layer.

3.3.3. Algorithm Step. This algorithm optimizes the RBF neural network, using subtractive clustering algorithm and improved the particle swarm optimization algorithm [26]. The following are the specific steps:

(1) Input the transmission frequency bands of a certain number of real frequency hopping signals (2000100 ) as set training samples optimized by the RBF neural network

(2) According to the subtractive clustering algorithm mentioned above, we perform clustering analysis on the samples, so that the number of centers can be determined

(3) Initialize the particle swarm, that is, we set the parameters of the PSO algorithm, as well as the initial position of the particles. The neurons in the hidden layer of the RBF neural network are represented by the particles here

(4) If the fitness of each particle is better than that of the best position it has ever experienced, then update the optimal position pbest ${ }_{i d}^{k}$ of the particle

(5) If the fitness of each particle is better than that of the best position experienced by the particle population, then update the global optimal position gbest ${ }_{i d}^{k}$ of the particle

(6) Modify the velocity and position of particles

(7) Continue the cycle from steps 4 to 6 and end the cycle when the result meets the calculation requirements

(8) Decode the best position experienced by the particle swarm and use the decoded value as the structural parameter of the RBF neural network. Then, learn from the network

\section{Performance Analysis}

In this experiment, 2000 data generated by MATLAB are used as training samples and 100 as test samples. The initialization values of the PSO-RBF algorithm are shown in Table 1.

Figures 4 and 5 compare between the real and predicted values obtained when the training set of different methods is 2000 data. There are 100 test sets, and it can be seen from the simulation figure that the distance between the real value 


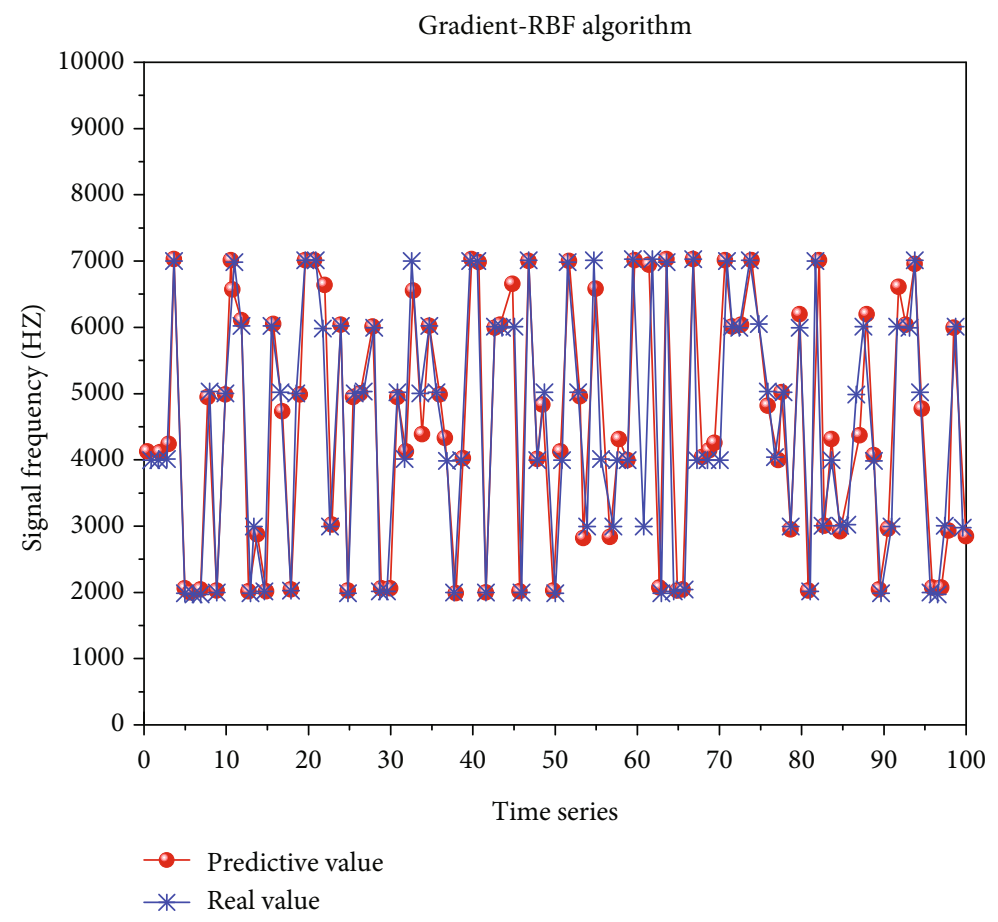

FIGURE 4: The error diagram of gradient-RBF prediction results.

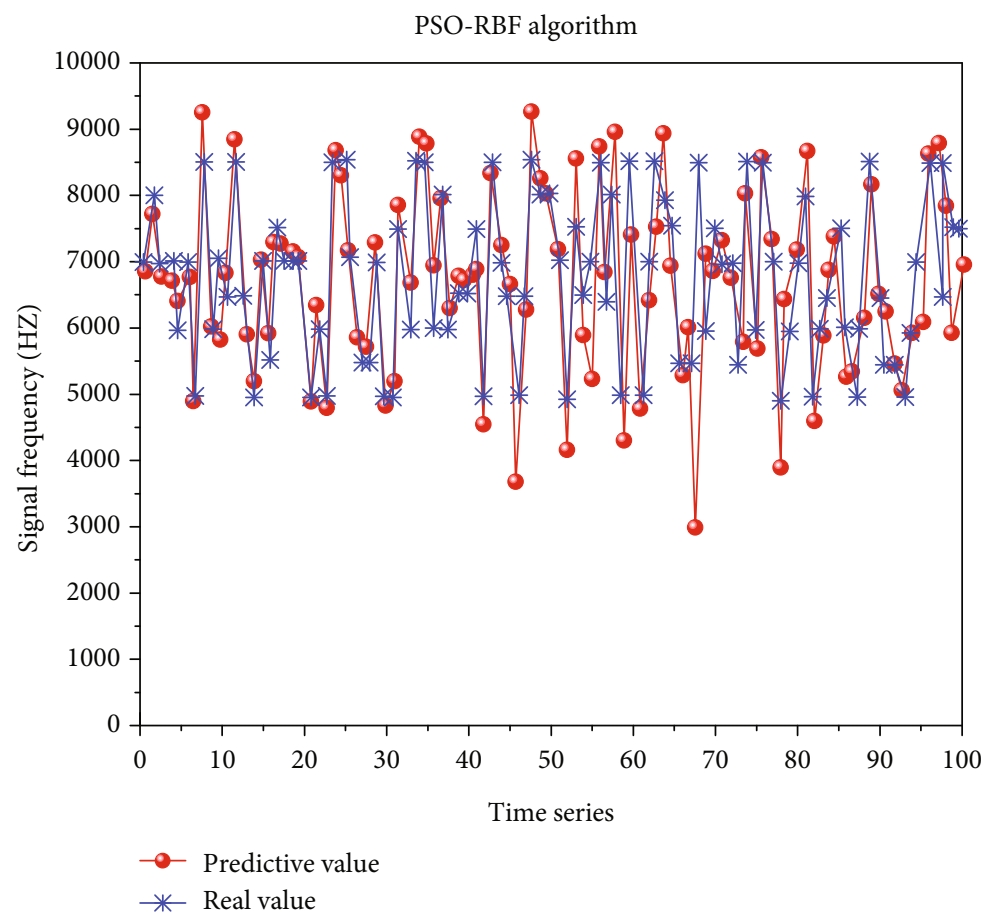

FIGURE 5: The error diagram of prediction result of PSO-RBF algorithms.

and the predictive value of most of the vast majority data is small, indicating that the error is small.

Because the scheme of this paper is mainly used to attack the enemy's communication system in the military field, therefore, the demand for time efficiency is high. In the first scheme, the RBF neural network based on the gradient descent method utilizes 100 to 2000 training data to analyze the impact of time efficiency and accuracy in detail. The details are shown in Figures 6 and 7 below.

Figure 7 depicts the changing trend between the gradientRBF algorithm and the traditional correlation-based algorithm model prediction accuracy and the simulation time 


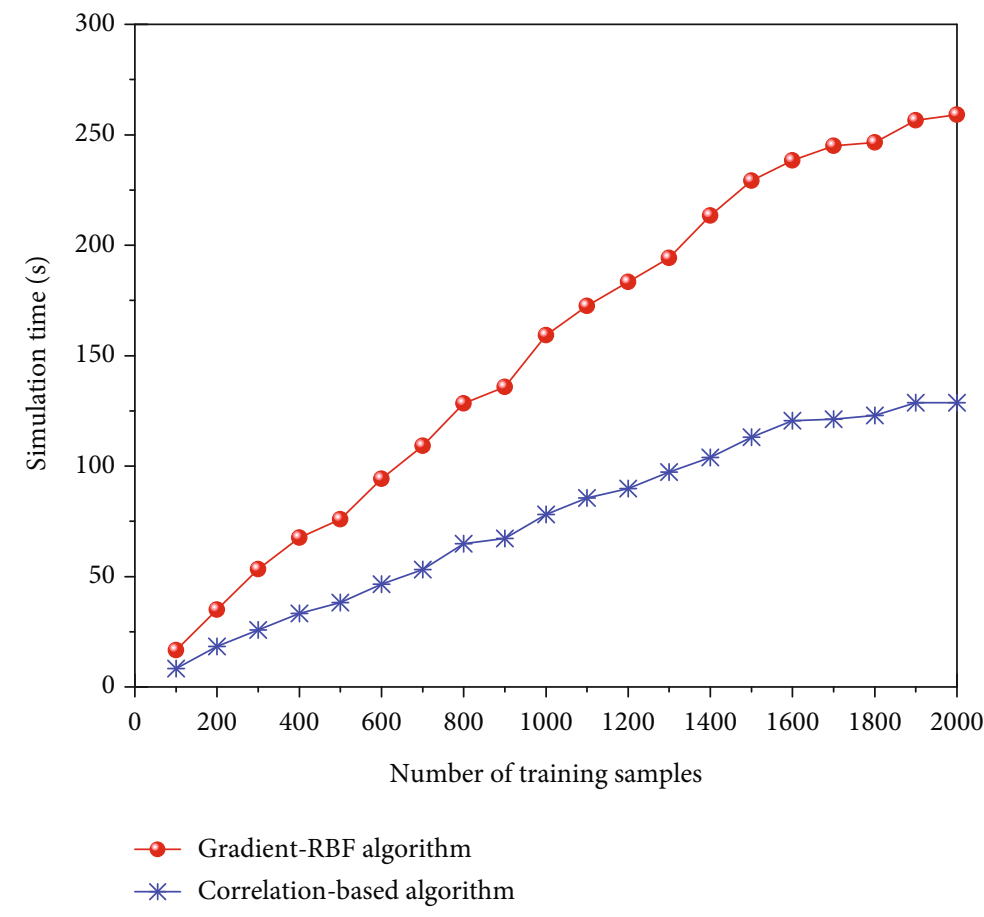

Figure 6: The relation diagram between the number of training samples and time efficiency.

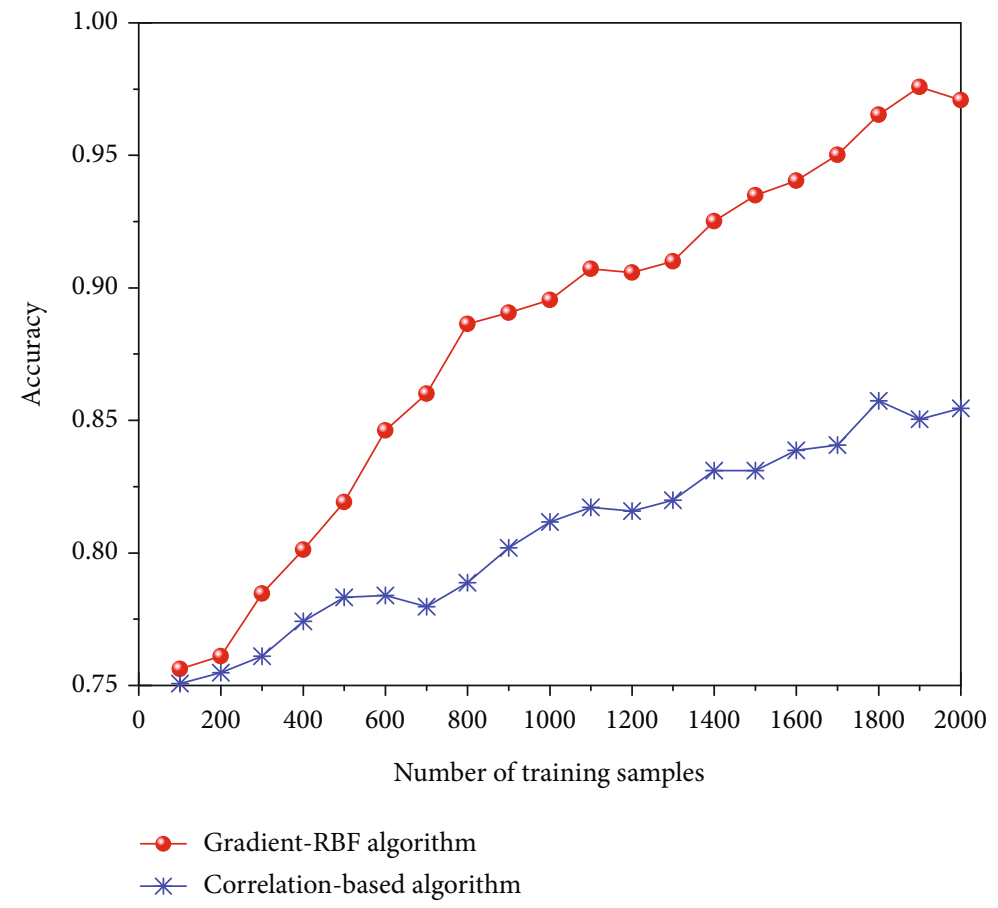

Figure 7: The accuracy comparison of the scheme.

of the algorithm. We can obtain from the figure that as the number of training sets increases, the simulation time curve of the two prediction models is tortuous. The simulation time of the gradient-RBF algorithm is getting longer. It can also be said that the simulation time is proportional to the number of training set samples. Compared with the simulation results of the two algorithms in the figure, the simulation time of the prediction model based on the correlation degree algorithm is significantly lower than that of the gradient-RBF algorithm. The cause of this trend is that the massive training samples and the massive iterations of the algorithm also increase, resulting in longer simulation times. 


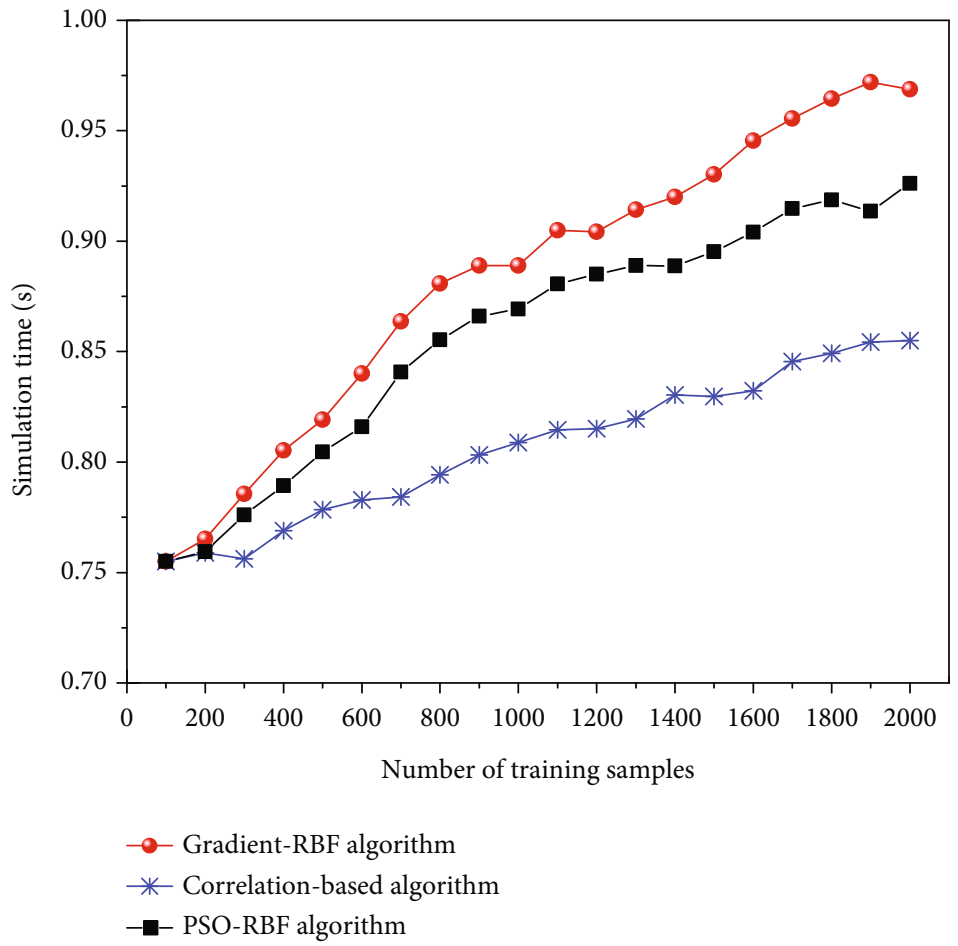

Figure 8: The relation diagram between the number of training samples and accuracy.

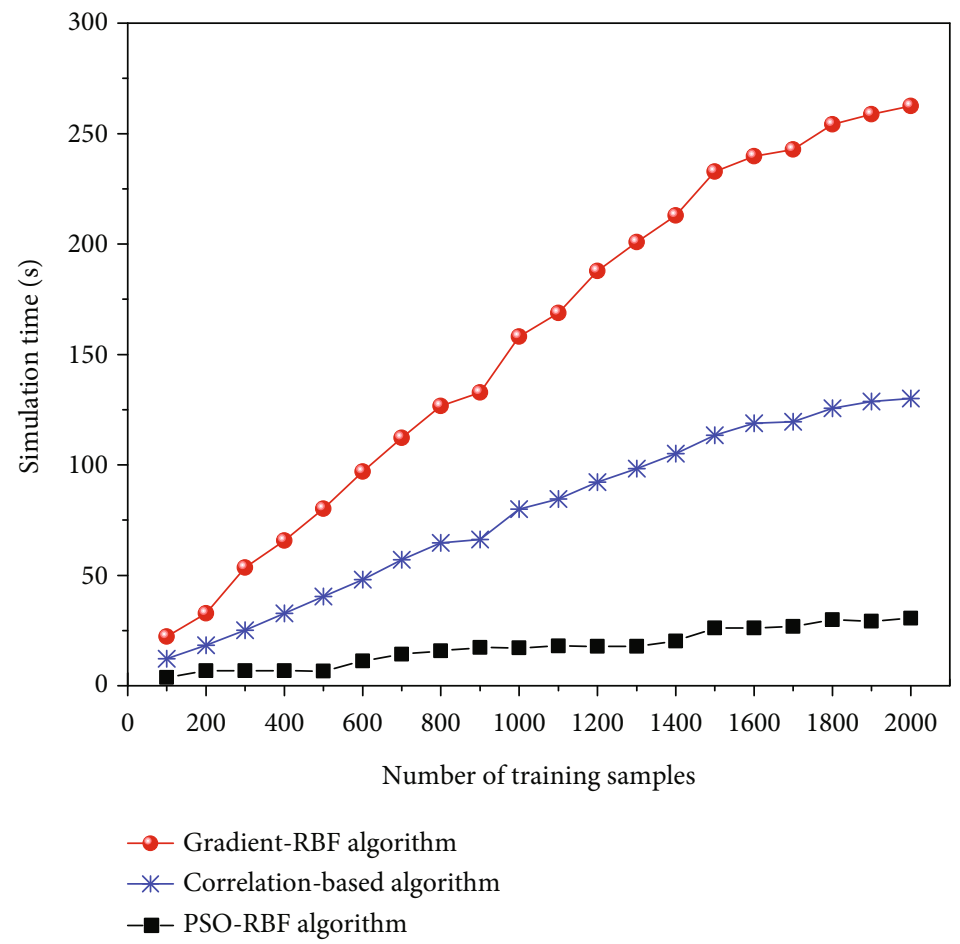

FIGURE 9: the relation diagram between the number of training samples and time efficiency.

Figure 8 shows the yellow line in the figure shows the changing trend between model prediction accuracy and accuracy in the PSO-RBF algorithm. Through the comparison of the three lines, we can see that the accuracy of the
gradient-RBF algorithm and the PSO-RBF algorithm is obviously better than the traditional correlation-based algorithm. As the number of training sets increases, the accuracy curve of the prediction model rises tortuously, and the accuracy 
of the algorithm gradually increases, up to $92 \%$. This indicates that the accuracy is proportional to the number of training set samples. The reason for this trend is that the number of training set samples increases, the number of iterations of algorithms also increases, and the center value and weight are better optimized, thus improving the accuracy of the algorithm. In this paper, we make use of the subtractive clustering algorithm to determine the number of centers of the radial basis function of the RBF neural network and the center value, width, and weight between the hidden layer and the output layer is optimized by the PSO algorithm. The experimental results illustrate that the optimization of the neural network by particle swarm optimization is effective.

Figure 9 shows that the yellow line in the figure indicates the changing trend between the model prediction accuracy of the PSO-RBF algorithm and the simulation time of the algorithm. Through the comparison of the simulation time of the three schemes, we can obtain that the simulation time curve of the prediction model rises tortuously as the number of training sets increases, and the simulation time of the algorithm becomes longer gradually. In other words, the simulation time is proportional to the number of training set samples. This is due to the number of training samples increasing, and the number of iterations of the algorithm also increasing, thus making the simulation time longer. The PSO-RBF algorithm of this scheme adopts the global optimization prediction model; so, the simulation time is much less than the other two schemes.

We select four specific indicators for systematic analysis to analyze the performance of the proposed scheme and the other existing schemes more clearly, including mean absolute error (MAE), mean absolute error rate (MAER), root mean square error (RMSE), and symmetrical mean absolute percentage error (SMAPE). The specific formulas are as follows. Through these indicators to judge the relationship between the final output and the actual value, the specific criteria are as follows:

(1) Mean absolute error (MAE) represents the actual situation of the prediction error

$$
\mathrm{MAE}=\frac{1}{n} \sum_{t=1}^{n}\left|X_{t}-F_{t}\right|,
$$

where $X_{t}$ is the actual value, $F_{t}$ is the predicted value, and $n$ is the total number of samples

(2) Mean absolute percentage error (MAPE) is a criterion for describing predicted and actual error values

$$
\mathrm{MAPE}=\frac{1}{n} \sum_{t=1}^{n}\left|\frac{X_{t}-F_{t}}{X_{t}}\right|,
$$

for MAPE, the closer the value is to 0 , the better the prediction effect is
TABLE 2: The quantitative analysis of 1000 training sets prediction results.

\begin{tabular}{lcccc}
\hline Algorithm & $\begin{array}{c}\text { MAE } \\
(\mathrm{Hz})\end{array}$ & MAPE & $\begin{array}{c}\text { RMSE } \\
(\mathrm{Hz})\end{array}$ & SMAPE \\
\hline $\begin{array}{l}\text { Gradient-RBF algorithm } \\
\begin{array}{l}\text { Correlation-based } \\
\text { algorithm }\end{array}\end{array}$ & 105 & 0.0361 & 11.7 & 0.0722 \\
PSO-RBF algorithm & 656 & 0.1452 & 24.6 & 0.01285 \\
\hline
\end{tabular}

TABLE 3: The error analysis of two schemes.

\begin{tabular}{lc}
\hline Algorithm & Simulation time (s) \\
\hline Gradient-RBF algorithm & 146 \\
Correlation-based algorithm & 82 \\
\hline
\end{tabular}

(3) Root mean squared error (RMSE) is the square root of the mean square difference between the prediction data and the original data

$$
\mathrm{RMSE}=\sqrt{\frac{1}{n} \sum_{i=1}^{n}\left(X_{t}-F_{t}\right)^{2}}
$$

(4) Symmetrical mean absolute percentage (SMAPE) is a criterion for describing predicted and actual errors, which is expressed as

$$
\mathrm{SMAPE}=\frac{2}{n} \sum_{t=1}^{n} \frac{\left|X_{t}-F_{t}\right|}{X_{t}+F_{t}}
$$

and the range of value is $[0,2)$. The closer the value is to 0 , the better the prediction effect and the smaller the error.

The real and predicted values of the test results of 1000 training in this scheme are brought into the above formulas. The result data can be obtained as follows.

In Table 2, SMAPE can clearly show the error between the real value and the prediction value of the system. The two schemes proposed in this paper are only slightly insufficient in accuracy compared with the gradient-RBF algorithm mentioned above when the data selected by the gradient-RBF algorithm is 1000 training sets.

This scheme compares the simulation running time of the two schemes through the specific data of Table 3, which directly shows the time defect of the gradient-RBF algorithm. The reason for this problem is that the number of iterations of the algorithm is too many.

It can be obtained from Table 4 that in the training process of the gradient algorithm, each iteration reaches the highest number of iterations, and the corresponding time is longer, while the prediction result is higher than that of the improved particle swarm algorithm. However, after meeting the minimum accuracy requirement, the improved particle swarm optimization algorithm does not continue to iterate. 
TABLE 4: The error-index analysis of two schemes of 2000 training sets.

\begin{tabular}{lcc}
\hline Algorithm & $\begin{array}{c}\text { Gradient-RBF } \\
\text { algorithm }\end{array}$ & $\begin{array}{c}\text { PSO-RBF } \\
\text { algorithm }\end{array}$ \\
\hline $\begin{array}{l}\text { Time performance } \\
\text { (s) }\end{array}$ & 256 & 28 \\
Accuracy & $98 \%$ & $92 \%$ \\
\hline
\end{tabular}

It optimizes the center, width, and weight of the RBF neural network from the global search. This scheme is much higher in time efficiency than the first scheme. As long as the parameter adjustment work is meticulous and the parameter adjustment is reasonable, the accuracy of the gradient algorithm can be achieved.

\section{Conclusions}

This paper analyzes and compares the features of related algorithms with their optimization algorithms. We apply each method to the frequency band prediction process of frequency hopping signals, compare the pros and cons of related algorithms, and propose two kinds of prediction schemes. The first is to first predict the center $c$ in the RBF neural network and use the cluster selection optimization algorithm. After optimizing the center $c$, using a gradient descent method and least mean square algorithm to calculate optimal learning rate, so as to improve the efficiency of the entire system. By analyzing simulation results, it is found that the accuracy of this scheme is remarkably progressed compared with a traditional correlation-based algorithm, but time efficiency is slightly longer. The second scheme is proposed in response to the shortcoming of the long computing time of the first scheme. It applies the improved particle swarm algorithm to the RBF neural network and uses the reduced clustering algorithm to calculate the number of RBF neural network centers. Then, the proposed scheme is used to directly optimize the center and weight for predicting the frequency problem of the frequency hopping signal of the nonlinear function in the RBF neural network. After the error analysis and comparison of the two schemes, it is found that using the second scheme to enhance the particle swarm optimization algorithm in the RBF neural network can effectively increase the time efficiency and make system accuracy above $90 \%$. In this way, it realizes the prediction of the nonlinear function, that is, the prediction of the frequency hopping signal, which makes it operable to attack the enemy's communication system in the specific military field. For our future work, we will consider blockchain-based communications and high-efficient learning solutions in our framework, such as $[27,28]$.

\section{Data Availability}

The dataset used in this paper is generated by MATLAB for evaluations.

\section{Conflicts of Interest}

The authors declare that there is no conflict of interest regarding the publication of this paper.

\section{Acknowledgments}

This work was supported in part by the National Natural Science Foundation of China under Grant 61931019, Shenzhen Technology Project (JCYJ20180302145648171), and the Fundamental Research Funds for the Central Universities (lzujbky-2020-kb25).

\section{References}

[1] W. Wang, F. Xia, H. Nie et al., "Vehicle trajectory clustering based on dynamic representation learning of internet of vehicles," IEEE Transactions on Intelligent Transportation Systems, pp. 1-11, 2020.

[2] J. Zhang, B. Wei, F. Wu et al., "Gate-ID: WiFi-based human identification irrespective of walking directions in smart home," IEEE Internet of Things Journal, 2020.

[3] C. Cormio and K. R. Chowdhury, "Common control channel design for cognitive radio wireless ad hoc networks using adaptive frequency hopping," Ad Hoc Networks, vol. 8, no. 4, pp. 430-438, 2010.

[4] W. Wang, X. Zhao, Z. Gong, Z. Chen, N. Zhang, and W. Wei, "An attention-based deep learning framework for trip destination prediction of sharing bike," IEEE Transactions on Intelligent Transportation Systems, pp. 1-10, 2020.

[5] W. Wang, T. Tang, F. Xia, Z. Gong, Z. Chen, and H. Liu, "Collaborative filtering with network representation learning for citation recommendation," IEEE Transactions on Big Data, pp. 1-14, 2020.

[6] Z. Ning, P. Dong, X. Wang et al., "Distributed and dynamic service placement in pervasive edge computing networks," IEEE Transactions on Parallel and Distributed Systems, vol. 32, no. 6, pp. 1277-1292, 2021.

[7] S. Gao, J. Sun, and X. Gao, "Soft-sensor modeling of rectification of vinyl chloride based on improved PSO-RBF neural network," in 2012 24th Chinese Control and Decision Conference (CCDC), Taiyuan, China, 2012.

[8] M. Er, S. Wu, J. Lu, and H. L. Toh, "Face recognition with radial basis function (RBF) neural networks," IEEE Transactions on Neural Networks, vol. 13, no. 3, pp. 697-710, 2002.

[9] J. Zhang, F. Wu, B. Wei et al., "Data augmentation and denseLSTM for human activity recognition using WiFi signal," IEEE Internet of Things Journal, vol. 8, no. 6, pp. 4628-4641, 2021.

[10] M. Xu, H. Chen, and L. Duan, "A combined training algorithm for RBF neural network based on particle swarm optimization and gradient descent," in 2020 IEEE 9th Data Driven Control and Learning Systems Conference (DDCLS), Liuzhou, China, 2020.

[11] X. Wang, Z. Ning, and S. Guo, "Multi-agent imitation learning for pervasive edge computing: a decentralized computation offloading algorithm," IEEE Transactions on Parallel and Distributed Systems, vol. 32, no. 2, pp. 411-425, 2021.

[12] W. Wang, F. Xia, J. Wu, Z. Gong, H. Tong, and B. D. Davision, "Scholar2vec: vector representation of scholars for lifetime collaborator prediction," ACM Transactions on Knowledge Discovery from Data, pp. 1-20, 2020. 
[13] X. Wang, Z. Ning, S. Guo, M. Wen, and V. Poor, "Minimizing the age-of-critical-information: an imitation learning-based scheduling approach under partial observations," IEEE Transactions on Mobile Computing, p. 1, 2021.

[14] J. Y. Chen, Z. Qin, and J. Jia, “A PSO-based subtractive clustering technique for designing RBF neural networks," in IEEE Congress on Evolutionary Computation (IEEE World Congress on Computational Intelligence), pp. 2047-2052, Hong Kong, China, 2008.

[15] Z. Ning, P. Dong, X. Wang et al., "Partial computation offloading and adaptive task scheduling for $5 \mathrm{G}$-enabled vehicular networks," IEEE Transactions on Mobile Computing, p. 1, 2020.

[16] B. Zhang, J. Y. Wang, and S. L. Zhang, "A new PSO-RBF model for groundwater quality assessment," Advanced Materials Research, vol. 463-464, pp. 922-925, 2012.

[17] Z. Ning, P. Dong, X. Wang et al., "Mobile edge computing enabled $5 \mathrm{G}$ health monitoring for internet of medical things: a decentralized game theoretic approach," IEEE Journal on Selected Areas in Communications, vol. 39, no. 2, pp. 463478, 2021.

[18] D. Xiang-Jun and W. Yan-Qin, "RBF neural network identifier based on optimal selection cluster algorithm and PSO algorithm and its application," in 2011 Fourth International Conference on Intelligent Computation Technology and Automation, pp. 884-887, Shenzhen, China, 2011.

[19] M. L. Zhang, "Ml-rbf: RBF neural networks for multi-label learning," Neural Processing Letters, vol. 29, no. 2, pp. 61-74, 2009.

[20] Z. Ning, S. Sun, X. Wang et al., "Intelligent resource allocation in mobile blockchain for privacy and security transactions: a deep reinforcement learning based approach," Science China Information Sciences, 2020.

[21] Y. Zhong, X. Huang, P. Meng, and F. Li, "PSO-RBF neural network PID control algorithm of electric gas pressure regulator," Abstract and Applied Analysis, vol. 2014, Article ID 731368, 7 pages, 2014.

[22] Z. Ning, K. Zhang, X. Wang et al., "Intelligent edge computing in Internet of Vehicles: a joint computation offloading and caching solution," IEEE Transactions on Intelligent Transportation Systems, pp. 1-14, 2020.

[23] G. Wenxian, W. Hongxiang, X. Jianxin, and Z. Yunfeng, "RBF neural network model based on improved PSO for predicting river runoff," in International Conference on Intelligent Computation Technology \& Automation, IEEE Computer Society, Changsha, China, 2010.

[24] Z. Ning, R. Y. K. Kwok, K. Zhang et al., "Joint computing and caching in 5G-envisioned internet of vehicles: a deep reinforcement learning based traffic control system," IEEE Transactions on Intelligent Transportation Systems, pp. 1-12, 2020.

[25] N. Zhang, "Urban stormwater runoff prediction using recurrent neural networks," in Advances in Neural Networks-ISNN 2011-8th International Symposium on Neural Networks, ISNN 2011, Guilin, China, 2011May 29-June 1, 2011, Proceedings, Part I. DBLP.
[26] J. Y. Chen and Z. Qin, Training RBF neural networks with PSO and improved subtractive clustering algorithms, pp. 1148-1155, Neural Information Processing, Berlin, Heidelberg, 2006.

[27] Z. Ning, S. Sun, X. Wang et al., Intelligent Resource Allocation in Mobile Blockchain for Privacy and Security Transactions: A Deep Reinforcement Learning Based Approach, SCIENCE CHINA Information Sciences, 2020.

[28] X. Wang, Z. Ning, S. Guo, and L. Wang, "Imitation learning enabled task scheduling for online vehicular edge computing," IEEE Transactions on Mobile Computing, p. 1, 2020. 